\title{
Fiber-coupled, high-Q AlGaAs microdisk cavities for chip-based cavity QED
}

\author{
Kartik Srinivasan, Paul E. Barclay, Matthew Borselli, Thomas J. Johnson, and Oskar \\ Painter \\ Thomas J. Watson, Sr. Laboratory of Applied Physics, California Institute of Technology, Pasadena, CA 91125, USA. \\ phone: (626) 395-6269, fax: (626) 795-7258, e-mail: kartik@caltech.edu \\ Andreas Stintz and Sanjay Krishna \\ Center for High Technology Materials, University of New Mexico, Albuquerque, NM 87106, USA.
}

\begin{abstract}
Fiber-coupled, high-quality-factor $\left(Q>10^{5}\right)$ AlGaAs microdisks with embedded quantum dots are demonstrated. Microdisk lasers employing evanescent fiber coupling for optical pumping and collection of emission exhibit improved efficiencies compared to devices probed through normalincidence, free-space photoluminescence.

(c) 2005 IEEE LEOS

OCIS codes: (230.5750) Resonators; (270.0270) Quantum Optics; (140.5960) Semiconductor Lasers
\end{abstract}

Cavity-QED-based devices operating in the strong coupling regime must meet relatively stringent requirements to maintain coherent interactions at the single quantum dot (QD), single-photon level. In particular, the strength of coupling between the quantum dot and the cavity field, $g$, must exceed the quantum dot dipole decay rate $\gamma_{\perp}$ and the cavity decay rate $\kappa$. In addition, an efficient method for injecting photons into the cavity and collecting emitted photons from the cavity is a requirement for applications in linear optics quantum computing and quantum networks. Here, we present results on quantum-dot-containing AlGaAs microdisks with cavity parameters that, under ideal conditions, satisfy the strong coupling condition $g>\left(\gamma_{\perp}, \kappa\right)$. Furthermore, optical fiber taper waveguides are used to provide an efficient coupling channel to the cavities, and photoluminescence measurements that utilize these tapers to improve the pump and collection efficiency from room-temperature, microdisk lasers are discussed.

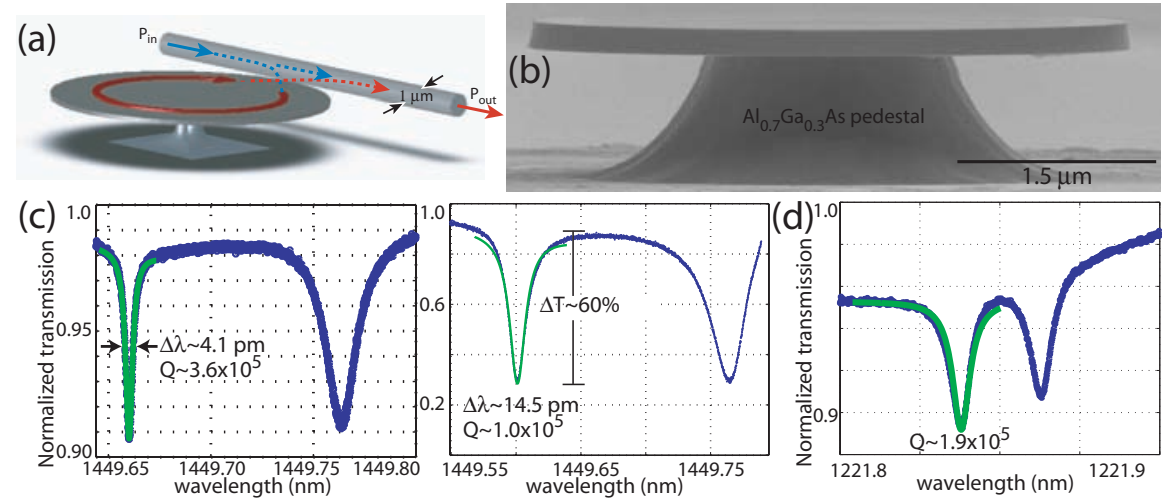

Fig. 1. (a) Schematic of the microdisk-fiber taper coupling geometry. (b) Scanning electron microscope images of a fabricated microdisk. (c) Normalized taper transmission $\left(P_{\text {out }} / P_{\text {in }}\right)$ in the $1400 \mathrm{~nm}$ wavelength band for large (left) and small (right) taper-cavity separations. (d) Normalized taper transmission in the $1200 \mathrm{~nm}$ band, $\sim 30 \mathrm{~nm}$ detuned from the peak QD emission.

Microdisk cavities (diameter $D=4.5 \mu \mathrm{m}$ ) are fabricated (Fig. 1(b)) in an AlGaAs-based waveguide containing a single layer of InAs quantum dots-in-a-well (DWELL)[1]. The DWELL material has its ground state emission peak at $1190 \mathrm{~nm}$, and direct passive measurements of optical loss in these structures is performed using an optical-fiberbased probing technique at wavelengths red-detuned from the QD emission (Fig. 1(a,c,d)). This method uses a tapered optical fiber to evanescently couple light to the whispering gallery modes (WGMs) of the microdisk. Starting with measurements in the $1400 \mathrm{~nm}$ band, where the DWELL absorption is expected to be minimal, a cold-cavity quality factor $(Q)$ of $3.6 \times 10^{5}$ is observed (Fig. 1(c,left)) for a WGM with a predicted effective modal volume $V_{\text {eff }} \sim 6(\lambda / n)^{3}$. By decreasing the taper-microdisk separation to $\sim 200 \mathrm{~nm}$, the coupling is increased to a depth $\Delta T \sim 60 \%$ for a loaded $Q \sim 1.0 \times 10^{5}$ (Fig. 1(c,right)), corresponding to a collection efficiency of emitted photons from the cavity of $\eta_{0} \sim 20 \%$. Furthermore, measurements in the $\lambda \sim 1.2 \mu \mathrm{m}$ band[2] show $Q \mathrm{~s}$ of $1.5-2.2 \times 10^{5}$ (Fig. 1(d)), indicating that high quality factors are still achieved despite the presence of some QD absorption and potential impurity states in the GaAs/AlGaAs epitaxy. Considering cavity QED applications, if these WGM modes with $\kappa / 2 \pi \lesssim 1 \mathrm{GHz}$ were optimally coupled to a single QD with a ground-state exciton spontaneous emission lifetime of $\tau_{s p} \sim 1 \mathrm{~ns}$ and low 
temperature homogeneous broadening of $\gamma_{\perp} / 2 \pi \sim 1 \mathrm{GHz}$, the corresponding coupling rate between the cavity mode photon and QD exciton would be $g / 2 \pi \sim 11 \mathrm{GHz}$, well within the strong coupling regime.

The high- $Q$ s demonstrated above indicate that room-temperature lasing can be achieved, and indeed, lasing has been observed in both pulsed and continuous wave operation[1], where the optical pumping and collection from the microdisks was performed at normal-incidence, through free-space optics. Here, we consider the use of fiber tapers in photoluminescence (PL) measurements of these devices[2]. Figure 2(a) shows the spectrum obtained in free-space PL measurements where a pump beam at $830 \mathrm{~nm}$ (spot size $\sim 18 \mu \mathrm{m}^{2}$ ) is focused upon a microdisk, and the emitted power is collected at normal incidence with a $0.4 \mathrm{NA}$ (numerical aperture) lens and resolved by an optical spectrum analyzer (OSA). Figure 2(b) shows the spectrum collected when a fiber taper is coupled to the side of the microdisk cavity, under identical free-space pumping conditions. The improved collection efficiency can be quantified by considering the differential efficiency of the device above threshold, $\xi$. In Figure 2(c)-(d), we see plot light-in-light-out (L-L) curves for lasing devices employing free-space and fiber taper collection, respectively. As expected (see Fig. 1(c), for example), the fiber taper loads the cavity and decreases its $Q$, causing the threshold pump power to (slightly) increase, but it also improves $\xi$, by nearly two orders of magnitude for this device, to a value of $\xi \sim 1 \%$ (when emission into the forward and backward fiber mode are considered). Modifications to the microdisk geometry through adjustments in thickness and radius can better match the effective index of the WGMs to that of the fundamental silica fiber taper waveguide mode, and can potentially increase the external fiber taper collection efficiency to $\eta_{0}>95 \%$.
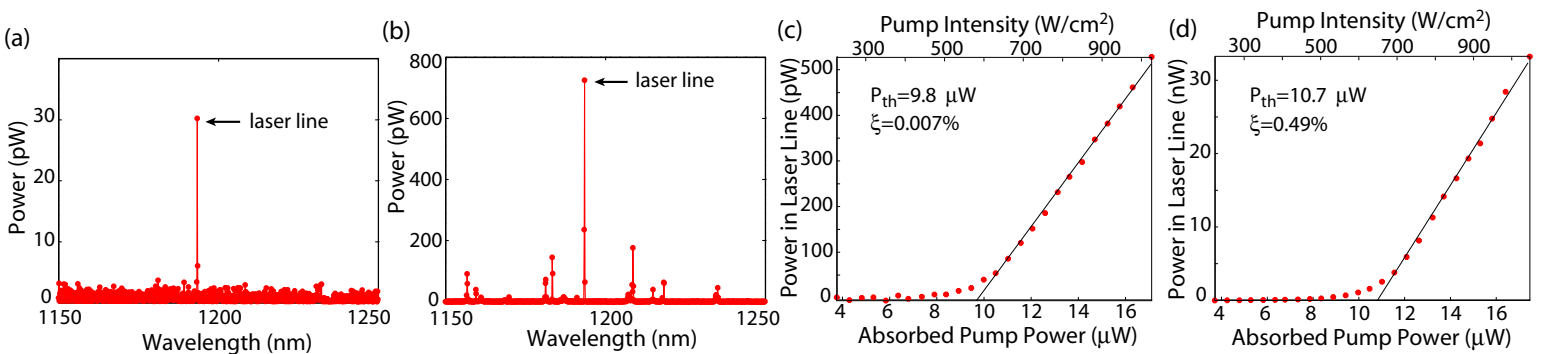

Fig. 2. (a)-(b) Collected spectrum in (a) free-space and (b) fiber taper configuration for a QD-containing microdisk that is pumped with $\sim 580 \mathrm{~W} / \mathrm{cm}^{2}$ of pump beam intensity. (c)-(d) Light-in-light-out (L-L) curves for the same device, where the light out is collected (c) free-space and (d) through the fiber taper. In (b) and (d), the fiber taper collected power included that from the forward propagating transmission channel only.

In addition, we have begun experiments utilizing the fiber paper as a means to inject pump light into the microdisks. Preliminary measurements indicate that significant reductions in the incident power delivered to the disk can be obtained when the pump wavelength is resonant with a WGM of the cavity. Microdisk lasers employing this fiber pumping and fiber collection have been demonstrated, and the results will be presented. The application of this work may ultimately lie in low-temperature experiments employing resonant pumping between states of single QDs. As such, progress towards incorporating fiber-coupled microcavity devices in cryogenic environments will also be discussed.

\section{References}

1. K. Srinivasan, M. Borselli, T. Johnson, P. Barclay, O. Painter, A. Stintz, and S. Krishna, "Optical loss and lasing characteristics of high-qualityfactor AlGaAs microdisk resonators with embedded quantum dots," Appl. Phys. Lett. 86, 151106 (2005).

2. K. Srinivasan, A. Stintz, S. Krishna, and O. Painter, "Photoluminescence measurements of quantum-dot-containing semiconductor microdisk resonators using optical fiber taper waveguides," submitted for publication (available at www.arxiv.org/abs/physics/0506105) (2005). 